\title{
INDICADORES SOCIALES Y VIOLENCIA DE GÉNERO: PROTECCIÓN DE LAS VÍCTIMAS Y CAMBIO SOCIAL EN LA UE
}

\author{
SOCIAL INDICATORS AND GENDER VIOLENCE: VICTIM PROTECTION \\ AND SOCIAL CHANGE IN THE EU
}

\author{
Gonzalo Montiel Roig \\ Universidad Jaume I. Castellón. España/Spain \\ gmontiel@,fis.uji.es
}

Recibido/Received: 13/04/2015

Modificado/Modified: 16/09/2015

Aceptado/Accepted: 04/10/2015

\section{RESUMEN}

El artículo realiza un análisis sobre los cambios jurídicos normativas de la UE en materia de violencia de género. En concreto, se ocupa del proceso de definición y categorización en el contexto de la reciente entrada en vigor de la "Orden europea de protección de las víctimas". En este sentido, el artículo realiza un análisis crítico sobre la construcción de indicadores y sobre la definición y coordinación de las políticas de la UE en materia de erradicación de la violencia de género y de atención a las víctimas. Desde esta óptica, el texto analiza las fuentes elaboradas por varias instituciones europeas sobre esta materia.

\section{PALABRAS CLAVE}

Violencia de género, políticas de igualdad, Estado de Bienestar, políticas europeas.

\section{SUMARIO}

1. Introducción. 2. Indicadores y fuentes comprables en la UE. 3. Asimetrías en la UE. 4. Políticas paliativas, políticas para el cambio social. 5. Conclusión. Bibliografía.

\begin{abstract}
This article is an analysis of the legal changes EU legislation on gender violence. Specifically, it deals with the process of defining and categorization in the context of the recent entry into force of the "European protection order for victims". In this sense, the article makes a critical analysis of the construction of indicators and the definition and coordination of EU policies on the eradication of gender-based violence and care for victims. From this perspective, the text analyzes the sources developed by several European institutions on this matter.
\end{abstract}

\section{KEYWORDS}

Gender violence, equality policies, Welfare State, European policies.

\section{CONTENTS}

1. Introduction. 2. Comparative indicators and sources at the EU on gender violence. 3. Asymmetries and welfare state in the EU. 4. Palliative policies and policies for social change. 5. Conclusion. References. 


\section{INTRODUCCIÓN}

Difícilmente se puede pretender investigar sin construir teórica y metodológicamente el objeto de estudio. Difícilmente se puede actuar sobre una realidad social que no se conoce. La Directiva 2011/99/ UE, de 13 de diciembre de 2011, sobre la orden europea de protección, y su transposición en el ordenamiento jurídico español a través de Ley 23/2014, de 20 de noviembre, de reconocimiento mutuo de resoluciones penales en la Unión Europea, es una aportación transcendental a la construcción de un marco jurídico europeo y también a la consecución de un marco de derechos, y por tanto, de igualdad real entre ciudadanas y ciudadanos europeos. Más allá de establecer un hito en la hoja de ruta definida, primero por el Tratado de Ámsterdam y luego en el Tratado de Lisboa para la creación de un espacio de libertad, seguridad y justicia en la Unión Europea y para la armonización de legislaciones y el reconocimiento mutuo de resoluciones judiciales, se trata de una herramienta que pondrá en evidencia, más si cabe, la necesidad de impulsar la cohesión de las políticas sociales y, por tanto, los derechos sociales de los ciudadanos que integran la UE. Este hito, que supone un paso más en la vertebración del proyecto político europeo, pone en evidencia también las lagunas en la coordinación y cohesión de los recursos y servicios que a cada uno de los ciudadanos y ciudadanas de la UE permite ejercer sus derechos y libertades en condiciones de igualdad. Pero también revela las desigualdades que todavía persisten en el seno de la UE. La orden europea de protección resuelve la necesidad de ejecutar, más allá de las fronteras y de los sistemas jurídicos del estado-nación, las medidas que han de aplicarse para proteger a las víctimas. La Directiva 2011/99/CE pone inevitablemente en evidencia la existencia de una diversidad de modelos de servicios y recursos públicos destinados a la extensión de derechos sociales, que implica también la existencia de una frontera interna que es todavía difícil de transitar, la frontera entre modelos del estados del bienestar. En definitiva, la puesta en marcha de una medida como la Orden europea de protección nos obliga a reflexionar y nos alerta sobre la existencia de diferentes modelos del estado de bienestar y de aplicación de los derechos sociales básicos.

A partir de esta primera reflexión, la cuestión que va a organizar este breve análisis sobre el contexto en el que se aplica la Ley 23/2014 de reconocimiento mutuo de resoluciones penales en la Unión Europea, la pregunta a la que tratamos de sacarle aristas, más que encontrar respuestas, en este trabajo es, ¿sobre qué realidad se aplica una iniciativa como la de la orden europea de protección? No se puede estudiar lo que no se conoce y no se puede describir aquello para lo que el lenguaje científico no se ha dotado de términos y categorías. No se puede intervenir sobre una realidad social a la que no se ha dotado de fuentes documentales, indicadores y mecanismos de análisis y prospección (Alcañiz, 2011:164); e incluso, disponiendo de esos recursos, corremos el riesgo de distorsionar y manipular la percepción de la realidad. Pero ese es un tema que, aunque nos afecta, ahora nos excede.

Las ciencias sociales sostienen sus aportaciones al conocimiento de la sociedad a partir de la construcción de un objeto de estudio que surge con la revolución industrial y las radicales transformaciones de las estructuras sociales de la modernidad y de la tecnificación de los procesos productivos y reproductivos con los que la especie humana acumula riqueza y poder. De la mano de esa necesidad por comprender, y también por intervenir, de manera racional en el conjunto de las transformaciones sociales, surge una nueva disciplina científico-social, la sociología, y un conjunto de paradigmas que 
aportarán conceptos, estructuras de pensamiento, formas de representar una sociedad en transformación. Ese objeto de describir, sondear y generar proyecciones de la sociedad está hecho de una materia compleja, de categorías, de constructos teóricos, de datos, de indicadores. Para ello, es necesario asumir institucional y científicamente ese empeño de definir el objeto de estudio para poder dar cuenta de una realidad que se intuye, pero que requiere de categorías y datos empíricos para ser interpelada e interpretada.

Como en el mito descrito por Platón, sabemos que la realidad existe, preconstruida como objeto de estudio por indicios, por sombras que se proyectan, en ocasiones mediatizadas por la influencia de las estructuras de poder, sobre los muros de la "caverna". El ecosistema comunicativo de un sociedad global, tecnificada y digitalizada es una enorme cúpula de sombras en la que se refleja, como en un continuo narrativo tejido de isotopías "greimasianas" coherentes y homogéneas, y espeluznantes en muchas ocasiones, el relato del machismo y del patriarcado generado por un magma mediático que busca garantizar la reproducción del modelo de relaciones sociales y de pareja y oculta la posible representación de un modelo alternativo (Montiel, 2009).

No se puede estudiar lo que no se conoce. No se puede describir aquello para lo que el lenguaje no se ha dotado de palabras. No se puede intervenir sobre una realidad social a la que no se ha dotado de fuentes documentales, indicadores y mecanismos de análisis y prospección (Alcañiz, 2011: 164); e incluso, disponiendo de esos recursos, corremos el riesgo de disponer de ellos para distorsionar y manipular la percepción de la realidad.

\section{INDICADORES Y FUENTES COMPARABLES EN LA UE}

La preocupación por la desigualdad de género y la voluntad decidida por erradicar la violencia de género no se ha constituido como problema central de las agendas de las políticas públicas hasta hace relativamente poco tiempo, y ello pese a que el proceso de modernización y racionalización que sostiene las sociedades modernas, centrales y europeas llevan tiempo describiéndose como tales en todos los órdenes de su identidad; y pese a que, como veremos, aun ahora, los indicadores, aunque han aumentado notablemente, siguen siendo limitados e insuficientes. Disponemos de herramientas estadísticas mucho mejores que las que teníamos hace sólo una década en España o en Europa, pero disponer de indicadores, no es disponer necesaria y absolutamente de un relato coherente de la realidad social, y tampoco supone tener todos los indicadores, ni todos los datos.

En los últimos años, las políticas de la UE han impulsado la institucionalización de la preocupación por el problema de la violencia de género y la desigualdad de género. Y como consecuencia, se ha hecho presente como objeto de estudio y de intervención político social. Es un hecho incontestable que el principio de igualdad está en la base de la constitución y el desarrollo de la UE, pero es también constatable que el desarrollo de sus políticas son fruto de culturas y estrategias sociales en algunos casos divergentes y contradictorias. Como ha destacado Lombardo (2014), en el proceso mismo de definición del modelo orgánico e institucional de aplicación de estas políticas y programas de igualdad, la UE ha puesto en evidencia diferencias y desencuentros muy importantes. Fruto de ello es la creación de dos organismos con enfoques diferentes pero dedicados ambos a objetivos relacionados con las políticas de igualdad en Europa, la European Union Agency for Fundamental Rights (en adelante FRA) y el European Institute for Gender Equality (en 
adelante EIGE). Las tareas asignadas a estos dos organismos y su impulso en materia de igualdad y género han sido esenciales en esa tarea de "institucionalizar" la construcción del objeto de estudio y de intervención social. Sin embargo, su constitución, su diferente enfoque del problema, en parte, es consecuencia, también, de la existencia de dos criterios diferentes en el seno de la UE: entre quienes promueven la necesidad de abordar los problemas relativos a las desigualdades de género en el seno de un programa que gestione de manera conjunta y holística las desigualdades y discriminaciones en los países de la UE, y quienes defienden la necesidad de impulsar un organismo que se centre en el análisis, impulso y supervisión de las políticas destinadas al fomento de la igualdad de género (Lombardo, 2014: 205-207). En cualquier caso, esta dualidad institucional, fruto de dos enfoques diferentes sobre cómo abordar la implantación y supervisión de las políticas de la UE, ha dado en poco tiempo importantes frutos y resultados para los investigadores y para los políticos, necesitados de referentes, indicadores, fuentes documentales y estadísticas sobre el mapa de las desigualdades de género y sus principales consecuencias y efectos en los diferentes estados de la UE.

En el caso del FRA, es necesario destacar la puesta en marcha de la macroencuesta sobre violencia contra las mujeres, basada en 42.000 encuestas personales a mujeres de 18 a 74 años mediante un extenso equipo de entrevistadores cualificados entre abril y septiembre de 2012 en los 28 estados miembros de la UE (alrededor de 1500 entrevistas por país). La encuesta ha analizado la experiencia personal de la violencia física, sexual, psicológica y económica, de acoso o persecución en mujeres desde los 15 años (European Union Agency for Fundamental Rights, 2014). Las conclusiones, extensas y complejas en su análisis e interpretación, revelan que una de cada tres mujeres ha experimentado violencia física o sexual, lo que supone un total de 62 millones de mujeres víctimas de este tipo de delito. Además, una de cada 10 ha experimentado alguna forma de violencia sexual y una de cada 20 ha sido violada. El estudio revela el alto nivel de vulnerabilidad de la mujeres jóvenes y el significativo porcentaje de violencia sufrida en el seno de la pareja: el $22 \%$ ha sufrido violencia por parte de su pareja, el $43 \%$ ha experimentado algún tipo de forma de violencia psicológica, y un 5\% ha experimentado violencia económica en sus relaciones personales (European Union Agency for Fundamental Rights, 2014: 9-39). Sin embargo, los datos que aporta esta amplia encuesta sirven, no sólo para establecer la dimensión del problema a escala europea, sino para trazar los ejes vertebradores de los retos de las políticas europeas en materia de políticas de género y de erradicación de la violencia de género. La encuesta aporta una información que, aislada de la realidad cultural y social de cada uno de los estados, con sus políticas y marcos legislativos y administrativos específicos, puede resultar parcial e incluso generar interpretaciones distorsionadas. De ahí que, pese a ser una fuente de información y de referencia, es necesario completar la definición del problema con otros indicadores.

$\mathrm{Y}$ en este sentido, el European Institute for Gender Equality (EIGE), a través de su proyecto Administrative data sources on $G B V$ in the EU (EIGE, 2014) ha aportado la creación de un complejo sistema de indicadores europeos sobre políticas de igualdad de género, entre las que se incluye, como una de las líneas estratégicas, la erradicación de la violencia contra la mujer como síntoma y obstáculo esencial en el desarrollo de cualquier política en este campo. El Gender Equality Index (EIGE, 2013) toma como referencia los datos aportados, entre otras fuentes, por la Gender Equality Unit del Directorate-General for Justice, la European Foundation for the Improvement of Living and Working Conditions (Eurofound), el European Women's Lobby, European Trade Union Confederation y las oficinas de datos estadísticos de los estados miembros. Esta aportación 
del EIGE al mapa europeo y global de la desigualdad de género enlaza con otros índices de referencia como el Gender-Related Development Index (GDI) mediante la agregación de datos de género al Human Development Index, o el Gender Empowerment Measure (Schüler, 2006; EWI, 2013; Council of Europe, 2014).

Sin embargo, el esfuerzo del EIGE en la construcción objetiva de la realidad social europea a través de este Gender Equality Index ha dejado, en su primer informe, un dato desalentador, y quizá también muy significativo, en el diagnóstico del problema de la violencia de género a nivel europeo: el EIGE, en el Gender Equality Index Report (2013), afirma no haber podido recuperar datos suficientes para construir un indicador sobre la situación de la violencia de género en la UE y establecer parámetros comparativos entre los diferentes estados:

"There are generally few indicators that can measure gender-based violence against women, since principles of crime classification systems for statistical use have yet to be established in the EU. The possibility of obtaining comparable administrative data on gender-based violence is also very limited at the EU level as there is no common methodology agreed within the Member States.

The norms, attitudes and stereotypes that largely underpin gender-based violence against women also remain critically under-measured, with, as a result, a strong dearth of potential indicators. There are to date no data sources that provide reliable, harmonised and comparable data for all Member States on these aspects.

$[\ldots]$

The extent to which the absence of indicators reflects gender relations should be questioned. The issue of what is being measured, or in this instance, not measured and why, are important questions. Therefore, the Gender Equality Index should be seen as a tool that calls for this data to be collected to fully measure gender equality within the framework of EU policy" (European Institute for Gender Equality, 2013: 141-142).

La falta de fuentes comparables y de indicadores que permitan evaluar el problema de la violencia de género a nivel europeo se convierte, de esta manera, en la principal conclusión y el eje central de reflexión del informe del EIGE. Y, más allá de la necesidad de construir esos indicadores, se abre el interrogante sobre su ausencia y sobre las razones últimas de la dificultad para confeccionarlos.

Las dificultades para abordar los indicadores sobre las dimensiones del problema de la violencia de género han sido puestas en evidencia también recientemente a partir de un estudio crítico de las macroencuestas elaboradas en España por el Instituto de la Mujer (1999, 2002 y 2006). El equipo de Elena Casado, Antonio A. García y Fernando J. García (2012), a partir de una réplica de la encuesta y mediante un análisis cualitativo de la recepción de los conceptos y categorías utilizados para confeccionar las preguntas, han destacado la necesidad de estandarizar y perfilar los conceptos con los que se interroga sobre la experiencia de la violencia de género a las mujeres encuestadas. Pero también revelan la dificultad para distinguir entre categorías como violencia y conflicto en las relaciones de pareja (2012: 184) o la pertinencia de incorporar en el análisis de la realidad, la percepción del problema entre la población masculina. 


\section{ASIMETRÍAS EN LA UE}

La aplicación de la Directiva 2011/99/ UE, sobre la orden europea de protección implica también, indirectamente y como ya hemos dicho, realizar una reflexión sobre la estructura de los servicios esenciales de apoyo social a las víctimas, como lo es la prestación de justicia gratuita, los servicios de acogida, atención e información, apoyo personal y social, etc. Sin la puesta a disposición de las mujeres que sufren la violencia de género de un conjunto de servicios y recursos necesarios para garantizar su protección integral, las medidas de protección jurídica pueden ser insuficientes o ineficientes. Algunas de las diferencias en materia de asistencia jurídica y de servicios en este ámbito pueden contrastarse en informes, como el Violence against Women. Victim Support (European Institute for Gender Equality, 2012), y, en especial, en los informes elaborados por Women Against Violence Europa (WAVE), en los que se evidencia las enormes diferencias en la cobertura de servicios básicos para la protección de las víctimas de las violencia de género, tanto por los que respecta al modelo de gestión, como por su capacidad de dar cobertura al conjunto de las victimas (Stelmaszek y Fisher, 2013; Lesur et al., 2014). En el Country Report 2013, el WAVE aporta un trabajo de investigación cualitativa, mediante la realización de 29 historias de vida. La conclusión de esta aportación revela un hilo conductor que marca todas las experiencias:

"A common theme observed in the stories is women's inability to escape the violence, due to lack of financial independence or lack of external support from family members. There is a certain shame associated with experiencing violence and women are often afraid to come forward to disclose the violence, feeling not only responsible for keeping the family unit together, but also fearing that they will be blamed or that they will not be believed or taken seriously by those to whom they report/disclose the violence" (Lesur et al., 2014: 11).

\section{POLÍticas PALIATIVAS, POLÍticas DE CAMBIO SOCIAL}

Una de las crítica al diseño actual de las políticas de igualdad de la UE se ha centrado en destacar el hecho de constituirse como un modelo paliativo y funcional, centrado en la integración laboral y productiva de las mujeres, y con pocas referencias o líneas específicas destinadas a incidir en los aspectos culturales y estructurales tanto de la desigualdad como de la violencia de género (Comisión de las Comunidades Europeas, 2000 y 2006; Comisión Europea, 2010; Consejo Europeo, 2000 y 2011). En este sentido, se ha destacado que la definición y diseño de las políticas de igualdad de género o de erradicación de la desigualdad se ha centrado de manera prioritaria en aspectos relacionados con la inserción laboral y con el desarrollo e integración de la mujer en la vida laboral y en los niveles de dirección y toma de decisiones en igualdad de condiciones con el hombre (Hafner-Burton y Pollack, 2002; Lombardo y Meier, 2008). Este eje central de las políticas, esencial por otra parte a la luz de los datos relativos a la desigualdad salarial y de representación en los cargos directivos de la mujer frente al hombre, ha llevado a las investigadoras Pastor Gonsálbez y Martín Herreros a hablar de una política en la UE en la que "prevalece el discurso utilitarista en el que se presenta como una pérdida de recursos humanos, la baja presencia de las mujeres en el mercado laboral y en puestos de poder" (2014: 180). Se trata, por tanto, de un enfoque, en el que tras la reivindicación de un trato igualitario de hombres y mujeres en el ámbito laboral se constituye en punto de 
partida para la recuperación de derechos y de empoderamiento femenino en la sociedad europea. Se trata de un argumento sostenido en un reconocimiento de las posibilidades del sistema para ajustar sus desequilibrios en el territorio de las necesidades productivas y reproductivas (Lombardo y Meier, 2008: 113). De igual manera, se ha puesto en evidencia en diversas ocasiones la falta de concreción en las medidas a aplicar para alcanzar los objetivos de igualdad real entre hombres y mujeres, así como la indeterminación de los indicadores de medición y el sistema de incentivos con el que se prevé un cambio de rumbo en el reparto de roles laborales que trascienda y transforme otras dimensiones de la sociedad (Pollack y Hafner-Burton, 2010: 292). Se estaría abordando, desde este punto de vista, los problemas derivados de la desigualdad de género o de la violencia contra las mujeres como un problema unidimensional, y no como un problema complejo, en el que los modelos de representación culturales refuerzan y reproducen las estructuras de la sociedad y sus desigualdades. De la misma manera, parece razonable que, tras la experiencia de la larga crisis del 2008, debería ser necesario abordar con una perspectiva compleja del problema de la desigualdad, en la línea del denominado enfoque "interseccional" descrito por Lombardo (2014: 199), y abordar la estructura de las desigualdades sociales como un enfoque afectado por múltiples variables y dimensiones de la desigualdad, como indica Verloo (2006: 214), en el que las desigualdades de género han de ser puestas en relación con otras desigualdades vinculadas a los conflictos internos y desigualdades preexistentes en la sociedad europea tales como las desigualdades marcadas por variables de territorio, de origen, de clase, cultura, religión o etnia.

Por otra parte, y en relación con el argumento anterior, en los documentos destinados a definir las políticas de igualdad de la UE en los últimos dos decenios se detecta una pérdida progresiva de la referencia al campo semántico de "cambio social", en los términos expuesto por investigadoras como Alcañiz (2004: 7-20), como el esfuerzo de las sociedades por impulsar cambios en las estructuras que reproducen y sostienen la desigualdad y la violencia estructural contra la mujer. Lo que no deja de ser la constatación de un giro permanente a lo largo del último decenio en la UE, en el que las políticas de corte neoliberal han ido ocupando espacio en el discurso europeo reconvirtiendo las bases del modelo de bienestar que acompañó en su origen el proyecto europeo y reorientando sus enfoques y neutralizando los objetivos sociales en sus planteamientos más transformadores, en la línea de lo que autoras como Simon-Kumar han descrito como giro post-liberal (2011: 442). A ello se ha referido también con insistencia Sarikakis al referirse a cómo las limitaciones para implantar las políticas de igualdad vienen acompañadas generalmente de argumentos resistentes que tienen que ver generalmente con el énfasis en la eficacia, los mecanismos internos del mercado y la necesaria limitación al intervencionismo en las estructuras dominantes en los organismos (2009: 204-205).

\section{CONCLUSIÓN}

En definitiva, como reflexión final, pese a los avances que supone la vertebración jurídico normativa de la UE a través de iniciativas como la Orden europea de protección, se percibe, como una constante que gana terreno en los poderes públicos occidentales y en las políticas de la UE, la pretensión de afrontar los problemas sociales como consecuencias de un cúmulo de decisiones individuales ajenas a los problemas estructurales del sistema. La construcción indicadores y la coordinación de sistemas de obtención de datos en la UE 
para la toma de decisiones políticas y para el diseño e implementación de las políticas de igualdad es una herramienta necesaria, y en la que se ha avanzado mucho en los últimos años. Pero la existencia de un mapa de datos e indicadores debe servir para constatar, no sólo problemas o disfunciones, sino también debe poner en evidencia las claves para definir, a corto o medio plazo, una hoja de ruta para el cambio social y la erradicación progresiva y definitiva de las desigualdades y la violencia de género, un problema estructural, cultural y multidimensional en la UE.

\section{BIBLIOGRAFÍA}

ALCAÑIZ MOSCARDÓ, M. (2011). Cambios y continuidades en las mujeres. Un análisis sociológico. Barcelona: Icaria.

ALCAÑIZ MOSCARDÓ, M. (2004). “Genealogía del cambio social”. RIPS, Revista de Investigaciones Políticas y Sociológicas, 3: 7-20.

CASADO APARICIO, E.; GARCÍA GARCÍA, A. A. y GARCÍA SELGAS, F. J. (2012). "Análisis crítico de los indicadores de violencia de género en parejas heterosexuales en España". EMPIRIA. Revista de Metodología de Ciencias Sociales, 24: 163-186.

COMISIÓN DE LAS COMUNIDADES EUROPEAS (2006). Plan de trabajo para la igualdad entre las mujeres y los hombres, 2006-2010 Bruselas, 1.3.2006 COM (2006) 92 final.

COMISIÓN DE LAS COMUNIDADES EUROPEAS (2000). Estrategia marco comunitaria sobre la igualdad entre hombres y mujeres (2001-2005) COM (2000) 335 final 2000/0143 (CNS).

COMISIÓN EUROPEA (2010). Estrategia para la igualdad entre mujeres y hombres 2010-2015.COM (2010) 491 final.

CONSEJO DE EUROPA (2014). Analytical study of the results of the fourth round of monitoring the implementation of Recommendations Rec (2002) 5 on the protection of women against violence in the Council of Europe member states. Accesible en: http://www.coe.int/t/dghl/standardsetting/conventionviolence/Docs/Analytical\%20Study\%20ENG.pdf (consulta 11/2/2015).

CONSEJO EUROPEO (2011). Pacto Europeo por la Igualdad de Género (2011-2020), (2011/C 155/02).

CONSEJO EUROPEO (2000). Decisión 2000/750/CE del Consejo, de 27 de noviembre de 2000, por la que se establece un programa de acción comunitario para luchar contra la discriminación (2001-2006) (DO L 303 de 2 de diciembre de 2000).

EUROPEAN INSTITUTE FOR GENDER EQUALITY, EIGE (2014). Administrative data sources on $G B V$ in the $E U$. Accesible en: http://eige.europa.eu/gender-based-violence/administrative-datasources\#country-data-info (consulta 11/2/2015). Y la fuente on line del Gender Equality Index http://eige.europa.eu/content/gender-equality-index\#/.

EUROPEAN INSTITUTE FOR GENDER EQUALITY (2013). Gender Equality Index Report. Accesible en: http://eige.europa.eu/apps/gei/content/Gender-Equality-Index-Report.pdf (consulta 11/2/2015).

EUROPEAN INSTITUTE FOR GENDER EQUALITY (2012). Violence against Women. Victim Support. Revieew of the Implementation of the Beijing Platform for Action in the EU Member States. Report. Luxemburgo: Publications Office of the European Union. Accesible en: http://eige.europa.eu/ sites/default/files/Violence-against-Women-Victim-Support-Report.pdf(consulta 11/2/2015).

EUROPEAN UNION AGENCY FOR FUNDAMENTAL RIGHTS, FRA (2014). Violence against women: an eu-wide survey - main results. Accesible en: http://fra.europa.eu/sites/default/files/fra-2014vaw-survey-main-results_en.pdf(consulta 11/2/2015).

EUROPEAN WOMEN'S LOBBY, EWl (2013) Rape Barometer. Accesible en: www.womenlobby. org/publications/rapports/article/2013-ewl-barometer-on-rape-report?lang=fr(consulta 11/2/2015).

HAFNER-BURTON, E. y POLLACK, M. A. (2002). "Mainstreaming Gender in Global Governance". European Journal of International Relations, 8: 339-373. 
LESUR, M.; STELMASZEK, B. y GOLDEN, I. (2014). Country report 2013. Reality Check on Data Collection and European Services for Women and Children Survivors of Violenc. A Right for Protection and Support? Viena: Women Against Violence Europa. Accesible en: http://www.wave-network.org/ content/wave-country-report-2013-now-online (consulta 11/2/2015).

LOMBARDO, E. (2014). "La 'intersección' de género en la política de la UE”. En M. I. Pastor Gonsálbez et al. (coords.) Integración europea y género. Madrid: Tecnos, pp. 199-218.

LOMBARDO, E. y MEIER, P. (2008). "Framing Gender Equality in the European Union Political Discourse". Social Politics: International Studies in Gender, State \& Society, 15: 101-129.

MONTIEL ROIG, G. (2009). “¿Qué contenidos generan violencia y desigualdad de género? Una aproximación a la regulación y la autorregulación de los medios de comunicación”. En J. Ma Bernardo Paniagua, E. Martínez García y G. Montiel Roig (coords. y eds.) Los retos de los medios de comunicación ante la violencia de género. Valencia: Tirant lo Blanch, pp. 115-135.

PASTOR GONSÁLBEZ, M. I. y MARTÍN HERREROS, V. (2014). "Las políticas de igualdad de género de la UE entre 2006 y 2012. Reflexiones críticas”. En M. I. Pastor Gonsálbez et al. (coords.) Integración europea y género. Madrid: Tecnos, pp. 179-198.

POLLACK, M. A. y HAFNER-BURTON, E. (2010). "Mainstreaming International Governance: The Environment, Gender, and IO Performance in the European Union". Review of International Organizations, 5: 285-313.

SARIKAKIS, K. y THAO NGUYEN, E. (2009). "The Trouble with Gender: Media Policy and Gender Mainstreaming in the European Union”. Journal of European Integration, 31(2): 201-216.

SCHÜLER, D. (2006). "The Uses and Misuses of the Gender-related Development Index and Gender Empowerment Measure: A Review of the Literature". Journal of Human Development, 7 (2): 161-181.

SIMON-KUMAR, R. (2011). "The Analytics of 'Gendering' in the Post-Neoliberal State. Social Politics: International Studies in Gender, State \& Society, 18 (3): 441-468.

STELMASZEK, B. / FISHER, B. (2013). Country report 2012. Reality Check on Data Collection and European Services for Women and Children Survivors of Violenc. A Right for Protection and Support? Viena: Women Against Violence Europa. Accesible en: http://www.wave-network.org/sites/ default/files/WAVE\%20COUNTRY\%20REPORT\%202012.pdf (consulta 11/2/2015)

VERLOO, M. (2006). "Multiple Inequalities, Intersectionality and the European Union". European Journal of Women's Studies, 13 (3): 211-228. 


\section{Breve currículo:}

\section{Gonzalo Montiel Roig}

Profesor asociado en el Departamento de Filosofía y Sociología de la Universidad Jaume I, y funcionario de la escala técnica superior de gestión cultural y patrimonio de la Universidad de Valencia Imparte asignaturas de teoría de la comunicación y estratificación social. Es miembro del Instituto Interuniversitario de Desarrollo Local y del Grupo de Investigación Multidisciplinar en Violencia de Género de la Universidad de Valencia. 